\title{
Managing chronic pain: are gabapentinoids being misused?
}

\author{
Tejus Anantharamu*,1 \& Mathur A Govind ${ }^{1}$ \\ ${ }^{1}$ Army College of Medical Sciences, Department of Pharmacology, New Delhi, India \\ *Author for correspondence: ddmtejus@gmail.com
}

First draft submitted: 29 April 2018; Accepted for publication: 8 May 2018; Published online: 13 September 2018

Keywords: chronic pain • gabapentinoids • misuse

Pain is a complex, multidimensional and unpleasant experience associated with emotional, cognitive, affective, behavioral and sensory components. The word 'pain' is derived from the Latin word 'poena' meaning penalty or punishment [1,2]. The International Association for the Study of Pain (IASP) in 1986 defined pain as "an unpleasant sensory and emotional experience which is primarily associated with tissue damage or described in terms of such damage, or both" [3]. Chronic pain is defined as persistent or recurrent pain lasting longer than 3 months [4].

Chronic pain has been categorized in the WHO 11th revision of International Classification of Diseases into seven groups namely chronic primary pain, cancer pain, post-traumatic and -surgical pain, neuropathic pain, headache and orofacial pain, visceral pain and musculoskeletal pain [4]. It affects approximately $20 \%$ of the population, accounting for $20 \%$ of physician visits worldwide. The prevalence of chronic pain varies, with WHO surveys estimating a prevalence of $37 \%$ in developed countries and $41 \%$ in developing countries [5]. Studies suggest that women, elderly, individuals with low income and those mentally ill are more likely to suffer from chronic pain [6].

\section{Managing chronic pain}

The American Pain Society had campaigned for making pain the fifth vital sign, in view of the enormity of suffering caused by chronic pain [6]. Treating chronic pain has always been challenging for clinicians and often needs a multidisciplinary approach $[6,7]$. A variety of drugs are available for the treatment of pain, which include NSAIDs, opioids, tricyclic antidepressants, serotonin noradrenaline reuptake inhibitors, corticosteroids, antiepileptics, antiarrhythmics, muscle relaxants, sedatives and anxiolytics [6]. Apart from pharmacotherapy, physical therapy like exercises, cognitive behavioral therapy and mindfulness-based stress reduction are often used to manage chronic pain $[6,7]$.

In 1986, the WHO has proposed a stepladder pattern for sequential use of analgesics, which includes NSAIDs for mild pain, followed by addition of mild opioids for moderate pain and strong opioids for severe pain [8]. Nonselective NSAIDs inhibit both COX enzyme 1 and 2, and inhibit prostaglandin production to relieve pain, but at the same time can cause various adverse effects like rash, dyspepsia, esophagitis, gastro-duodenal ulcers, exacerbations of asthma, seizures, dizziness, confusion, acute renal failure, heart failure and stroke [8,9]. While the selective COX-2 inhibitors like etoricoxib, valdecoxib and celecoxib, display the same efficacy and improved gastric tolerance compared with nonselective COX inhibitors, but result in an increased risk of edema, hypertension, myocardial infarction and stroke, due to inhibition of prostacyclin (PGI2) synthesis, without having much effect on thromboxane A2 (TXA2) synthesis $[8,9]$.

Opioid analgesics are generally reserved for moderate to severe pain as their use is associated with various adverse effects like sedation, nausea, vomiting, constipation, urticaria, urinary retention, respiratory depression, seizures, tolerance, dependence, abuse, hypogonadism and immunosuppression $[8,10]$. The rising incidence of chronic pain had forced physicians to use opioids for the same which heralded the epidemic of opioid dependence, currently discussed in numerous academic forums [11,12]. This unique situation of the need for tackling pain and preventing opioid dependence has encouraged the exploration of other alternatives for managing chronic pain [11,12]. 


\section{Role of gabapentinoids in managing chronic pain}

Although a number of drug classes are available, each has its own limitations. In this scenario gabapentinoids (gabapentin and pregabalin) are often used as nonopioid and non-NSAID options, which are recommended as firstline agents for neuropathic pain [12]. Gabapentinoids are Gamma Aminobutyric Acid (GABA) derivatives approved as adjunct antiepileptics. Gabapentin reduces calcium entry into presynaptic neurons by modulating $\alpha 2 \delta 1$ subunits of the voltage-gated calcium channels, thereby reducing glutamate release and reducing neuronal excitability. It is unclear whether the analgesic action of gabapentin is due to its GABA releasing or calcium channels modulating action [13]. It has been approved by the US FDA for postherpetic neuralgia in 2002 and widely used for the same [14]. Pregabalin, a congener of gabapentin has been approved for use in postherpetic neuralgia (2004), neuropathic pain associated with diabetes or spinal cord injuries (2004) and fibromyalgia (2007) [12,14].

Gabapentinoids were promoted extensively by their manufacturers for various off-label uses including trigeminal neuralgia, migraine, bipolar disorders, restless leg syndrome and various pain syndromes [12,14,15]. A trend of increase in prescriptions for gabapentinoids has been reported by many researchers in their studies [12,14,15]. An estimate suggests that $>95 \%$ of the prescriptions of gabapentinoids are off label in nature [12,15]. This becomes important in the backdrop of many researchers as well as several national and international pharmacovigilance databases reporting abuse liabilities and overdose fatalities with gabapentinoids [14,15].

\section{Review of literature}

Evoy KE et al. in their systematic review found sufficient evidence to suggest that gabapentinoids have the potential to be abused and estimated a prevalence of $1.6 \%$ in general population, whereas the same ranges between 3 and $68 \%$ among abusers of opioids [16].

Smith RV et al. in their systematic review on gabapentin misuse, abuse and diversion have brought out the reasons for misuse of gabapentinoids to be either for recreation, self harm or as self medication. Gabapentinoids are known to cause a wide range of subjective symptoms including euphoria, sedation and dissociation, which can contribute to their misuse, but the dose range or the therapeutic plasma concentration causing the same is yet to be defined [17].

Gomes $\mathrm{T}$ et al. in their population-based nested case-control study observed that $8 \%$ of the patients receiving opioids were co-prescribed gabapentinoids and this was associated with a $50 \%$ increase in the risk of opioid-related deaths, whereas no such association was observed between opioids and NSAIDs co-prescription. They postulated that the reasons for the same were pharmacokinetic and pharmacodynamic interactions between opioids and gabapentinoids. Opioid-induced reduced gastrointestinal motility could enhance the absorption of gabapentinoids leading to their enhanced plasma concentration, which could have an additive respiratory depression [18].

\section{Conclusion}

Although the prevalence of misuse or abuse of gabapentinoids appears to be minimal at present, the same cannot be neglected. The alarming trend of opioid dependence resulting from their use in the management of chronic pain is likely to act as a catalyst for increasing the same. Further monitoring of the situation might bring out more cases of gabapentinoid misuse or abuse. Till the situation becomes clear and regulatory authorities bring out suitable guidelines in respect of gabapentinoid use in pain syndromes, it would be wise on the part of clinicians to exercise caution so as to not further fuel the off-label use of gabapentinoids for various pain syndromes.

\section{Financial \& competing interests disclosure}

The authors have no relevant affiliations or financial involvement with any organization or entity with a financial interest in or financial conflict with the subject matter or materials discussed in the manuscript. This includes employment, consultancies, honoraria, stock ownership or options, expert testimony, grants or patents received or pending, or royalties.

No writing assistance was utilized in the production of this manuscript.

\section{References}

1. Jagadeesh K. Comparison of intrathecally administered fentanyl and midazolam in combination with hyberbaric bupivacaine. J. Evid. Based Med. Healthcare 2(24), 3587-3595 (2015).

2. Gugsa N G. History pain and pain management. Res. Med. Eng. Sci. 3(4), 1-3 (2018).

3. Andreu V, Arruebo M. Current progress and challenges of nanoparticle based therapeutics in pain management. J. Control. Release 269, 189-213 (2018). 
4. Treede R-D, Rief W, Barke A et al. A classification of chronic pain for ICD-11. Pain 156(6), 1003-1007 (2015).

5. Fayaz A, Croft P, Langford RM, Donaldson LJ, Jones GT. Prevalence of chronic pain in the UK: a systematic review and meta-analysis of population studies. BMJ Open 6, e010364 (2016).

6. Tompkins AD, Hobelmann G, Compton P. Providing chronic pain management in the 'Fifth Vital Sign' era: historical and treatment perspectives on a modern-day medical dilemma. Drug Alcohol Depend. 173(1), S11-S21 (2017).

7. Joshi GM. Holistic care in chronic pain. Indian J. Pain 31, 77-79 (2017).

8. Ussai S, Miceli L, Pisa FE et al. Impact of potential inappropriate NSAIDs use in chronic pain. Drug Des. Devel. Ther. 9, 2073-2077 (2015).

9. Crofford LJ. Use of NSAIDs in treating patients with arthritis. Arthritis Res. Ther. 15(Suppl 3), 2-10 (2013).

10. Yaksh TL, Wallace MS. Opioids, analgesia, and pain management. In: Goodman \& Gilman's The Pharmacological Basis of Therapeutics (12th Edition). Brunton LL, Chabner BA, Knollmann BC (Eds). McGraw-Hill, CA, USA, 81-527 (2011).

11. Rosenblatt RA, Catlin M. Opioids for chronic pain: first do no harm. Ann. FAM Med. 10(4), 300-301 (2012).

12. Goodman CW, Brett AS. Gabapentin and pregabalin for pain - is increased prescribing a cause for concern? N. Engl. J. Med. 377 , 411-414 (2017).

13. Patel R, Dickenson AH. Mechanisms of the gabapentinoids and $\alpha_{2} \delta-1$ calcium channel subunit in neuropathic pain. Pharmacol. Res. Perspect. 4(2), e00205 (2016).

14. Morrison EE, Sandilands EA, Webb DJ. Gabapentin and pregabalin: do the benefits outweigh the harms? J. R. Coll. Physicians Edinb. 47(4), 310-313 (2017).

15. Wallach JD, Ross JS. Gabapentin approvals, off-label use, and lessons for post-marketing evaluation efforts. JAMA 319(8), 776-778 (2018).

16. Evoy KE, Morrison MD, Saklad SR. Abuse and misuse of pregabalin and gabapentin. Drugs 77(4), 403-426 (2017).

17. Smith RV, Havens JR, Walsh SL. Gabapentin misuse, abuse, and diversion: a systematic review. Addiction 111(7), 1160-1174 (2016).

18. Gomes T, Juurlink DN, Antoniou T, Mamdani MM, Paterson JM, van den Brink W. Gabapentin, opioids, and the risk of opioid-related death: a population-based nested case-control study. PLoS Med. 14(10), e1002396 (2017). 
